\title{
INTERACTIVE CLASSIFICATION OF CONSTRUCTION MATERIALS: FEEDBACK DRIVEN FRAMEWORK FOR ANNOTATION AND ANALYSIS OF 3D POINT CLOUDS
}

\author{
M. R. Hess ${ }^{\mathrm{a}, *}$, V. Petrovic ${ }^{\mathrm{b}}$, F. Kuester ${ }^{\mathrm{a}}$ \\ ${ }^{a}$ Dept. of Structural Engineering, University of California, San Diego, USA - (mrhess, fkuester)@ucsd.edu \\ ${ }^{\mathrm{b}}$ Dept. of Computer Science and Engineering, University of California, San Diego, USA - vipetrov@ ucsd.edu
}

Commission VI, WG VI/4

KEY WORDS: Point Cloud, Visualization, Interactive, Annotation, Classification

\begin{abstract}
:
Digital documentation of cultural heritage structures is increasingly more common through the application of different imaging techniques. Many works have focused on the application of laser scanning and photogrammetry techniques for the acquisition of threedimensional (3D) geometry detailing cultural heritage sites and structures. With an abundance of these 3D data assets, there must be a digital environment where these data can be visualized and analyzed. Presented here is a feedback driven visualization framework that seamlessly enables interactive exploration and manipulation of massive point cloud data. The focus of this work is on the classification of different building materials with the goal of building more accurate as-built information models of historical structures. User defined functions have been tested within the interactive point cloud visualization framework to evaluate automated and semi-automated classification of 3D point data. These functions include decisions based on observed color, laser intensity, normal vector or local surface geometry. Multiple case studies are presented here to demonstrate the flexibility and utility of the presented point cloud visualization framework to achieve classification objectives.
\end{abstract}

\section{INTRODUCTION}

Laser scanning and photogrammetry techniques have been used extensively for the acquisition of three-dimensional (3D) geometry of cultural heritage sites and structures. Existing structures, especially historical buildings which have existed for centuries, have undergone changes including additions, floor plan modifications, and physical alterations to original building materials. One crucial step to take before making assessments or decisions concerning preservation or restoration is to document what currently exists. Photogrammetry and laser scanning provide initial geometric information that can be visualized and analyzed, and these data can also serve as a scaffold for derivative data layers. Just as an architect would traditionally annotate an engineering drawing of a building, these 3D models should be annotated to provide additional information to aid in the interpretation of the structures state of health as well as decisions concerning its future. A natural first step in this process is to classify the structures construction materials into different categories.

Presented here is a feedback driven visualization framework (Petrovic et al., 2014, Petrovic et al., 2011) that seamlessly enables interactive exploration and manipulation of massive point cloud data (Figure 1). There are countless objectives that can be achieved through the acquisition, visualization and analysis of point clouds. The focus of this work is on the classification of different building materials with the goal of building more accurate as-built models of historical structures. Varying user defined functions will be tested within the interactive point cloud visualization framework to evaluate automated and semi-automated classification of 3D point data. These functions can include decisions based on observed color, laser intensity, normal vector or local surface geometry.

\footnotetext{
${ }^{*}$ Corresponding author
}

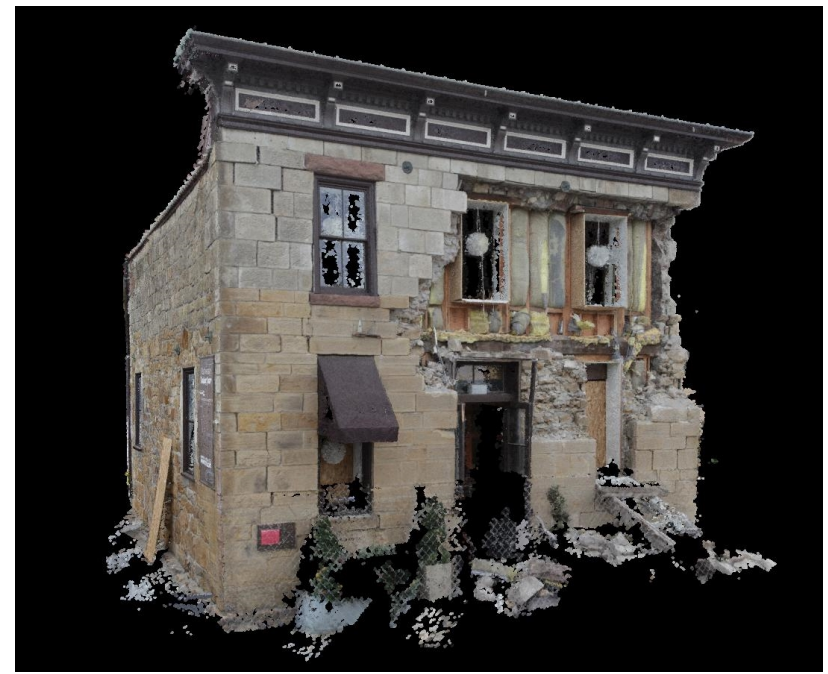

Figure 1. Photogrammetric point cloud in visualization engine

Automatic classification of point cloud features is not always an accurate or straightforward process. The presented approach leverages interactive user inputs in order to classify building geometry more accurately and efficiently. Users have the ability create their own functions in JavaScript and the functions will be executed on top of the point cloud engine. The visualization system handles the organization and queries to the point data and the users function will execute on the identified set of points. With the ability to manipulate and analyze billions of points in real time on available hardware, the visualization framework enables the swift addition of metadata to the 3D geometric model. The raw point data are not altered; instead, tags are associated with the points with the ability for a point to have multiple tags as well 
as dynamically changing tags. The operating scenario considers a field expert as the user and the functions are designed by the user to achieve their objectives. Utilizing the experts guided input, the system then performs automated routines to refine the classification. This semi-automated hybrid approach to classification allows more complicated features to be extracted and annotated through interactive feedback. The visual feedback achieved through the visualization system, allows for the user to more accurately refine the functions being executed to optimize the automated portion of the process.

Previous works have discussed the benefits of these 3D data assets for documentation objectives, measurements, and quantified analyses (Pesci et al., 2012, Guidi et al., 2014, Hess et al., 2015, Volk et al., 2014). Explored here is how to bridge the gap from digital documentation towards Historical Building Information Models (HBIM) (Quattrini et al., 2015, Oreni et al., 2014) that not only capture the current state of a structure, but also break down the virtual structure into its components. A vast majority of historical structures that still exist today are masonry constructions, so the focus of this work is to achieve the classification of the observed masonry materials through the analysis of 3D data derived from laser scanning or photogrammetric techniques.

Multiple examples are presented here to demonstrate the flexibility and utility of the presented point cloud visualization framework to achieve classification objectives. The presented datasets were generated through laser scanning, photogrammetry, or a combination of the two techniques. The included examples focus on the classification of construction materials used in cultural heritage structures. Specifically this work demonstrates multiple approaches to automatically and semi-automatically classify masonry structures into their stone and mortar counterparts. The accurate geometric classification of existing masonry materials will aid in more realistic numerical simulations of a structure in order to plan for preservation and restoration actions.

Observations that can be quantified in this case study include the average sizes of masonry units and mortar joints, variation and distribution of those dimensions, in addition to overall masonry patterning. The goal of this study is to discourage general assumptions that can be made concerning the local geometry and uniformity of masonry constructions. In other words, highlighted here is a methodology to capture the as-built structural state. Although, in some cases, assumptions concerning masonry geometry may not lead to vastly different conclusions, the capability to accurately document the geometry exists and should become standard practice in order to more precisely assess a structures state of health.

\section{BACKGROUND}

Digital documentation has been widely implemented in the field of cultural heritage and there are many applications of the derivative data. The focus here is on the utility of three-dimensional (3D) data acquired at cultural heritage sites. The uses for these data have elevated from simple documentation for posterity (Ikeuchi et al., 2003, Guarnieri et al., 2004) all the way to numerical structural simulations of entire complex structures (Crespi et al., 2015). The idea of Historic building information modelling (HBIM) has been developed (Murphy et al., 2009) with the idea of gathering survey data with remote imaging techniques in order to build 3D models that capture the geometry of the existing structure along with other types of information. Building information models
(BIM) are typically used for new construction, but there is a significant benefit in using it for preservation and restoration purposes.

Previous works have started to add intelligence to these types of HBIM models through recognition and modeling of architectural and structural components (Murphy et al., 2013, Oreni et al., 2014, Dore and Murphy, 2013). The creation of HBIMs can aid in the creation of construction drawing, documentation of existing damages, planning restoration activities, and eventually finite and discrete element modeling for the most accurate numerical simulation of existing structures. The two major gaps that this work looks to address are: 1) the simplification of geometric irregularities and 2) the 3D classification of masonry construction. Through the implementation of user guided annotation, dense 3D point clouds can be classified interactively both manually and semi-automatically.

Previous works have attempted masonry classification from a few different approaches. There are many that have used image processing techniques to isolate masonry stones from mortar (Del Pozo et al., 2015, Dimitrov and Golparvar-Fard, 2014), but there can be issues due to lighting, irregular stone shapes and patterns as well as varying construction phases and styles. Others have utilized laser scanning techniques with the attempt of overcoming some of the problems encountered when simplifying the problem to two dimensions (Sithole, 2008, Riveiro et al., 2016). Even when using 3D data there are difficulties to overcome when attempting to automate classification of a construction technique that varies significantly in time, region, and materials.

Explored here is how to build Historical Building Information Models that break down the virtual structure into its components and maintain the complexity of an existing historical structure. In order to preserve the irregularities and complexities, point cloud data is utilized in order to maintain a superior level of accuracy detail as compared to meshing methods. The interactive nature of the presented approach provides real-time feedback to the user in order to more accurately classify the construction materials and fine tune the classification methods through user customizable JavaScript code.

\section{PRESENTED APPROACH}

The goal of the presented approach is to utilize valuable user intuition and input in order to classify 3D masonry material faster and more accurately. The approach uses a "paint brush", as seen in Figure 2, that interactively operates on a selected sphere of points within the point cloud. The size of the sphere used to query the points is adjustable and can be changed dynamically. The operations that are performed within the brushing sphere are designed through a real-time updated JavaScript framework that communicates with the visualization engine in order to pass and execute the point data queries. Attributes of the points such as coordinates, color, normal and intensity can each be accessed and utilized in the JavaScript functions.

Through this flexible visualization system the user can dynamically annotate extremely large point clouds in real-time. The ability to manipulate the model in 3D space makes the job of annotating the model much easier with the ability to alter ones perspective with ease. The example model used, as seen in Figure 1, is a point cloud derived through photogrammetric processing of images in order to render a geometric model. This structure was 

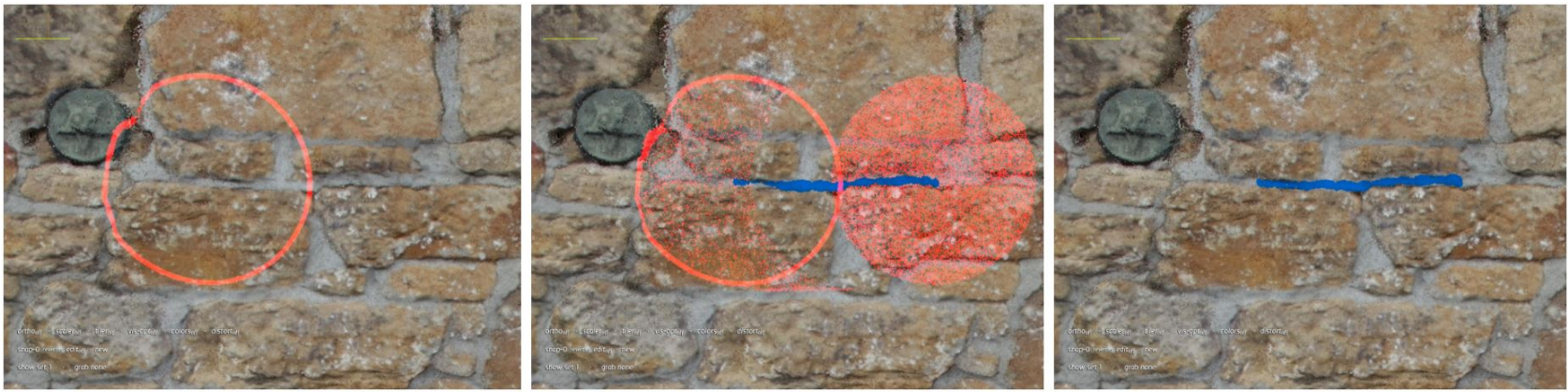

Figure 2. Example of the interactive annotation used on dense point cloud

used due to the different phases of construction as well as the varying styles and colors used in the execution of the masonry facade.

The first step of the presented example was to create a ground truth to compare our semi-automated tests against. The mortar in the front and the side of the building were manually annotated, or tagged, with pink and green respectively (seen in Figure 3). This ground truth tagging has three areas of style and color that each present their own challenges. The lower portion of the side wall is very irregular in pattern, constructed with stones that have a very different color than the mortar material and the topography of the mortar is very well defined. The topography of the mortar in the upper portion of the side wall is also reasonably well defined, but the colors of the stone and mortar are very similar. As for the front of the building, the construction is very regular with small mortar joints that as similar in color and do not have a very pronounced geometric pattern. Even though this ground truth creation required two and a half hours, the interactive manipulation of the point cloud made it extremely easy to select neighborhoods of points corresponding to mortar. The resulting annotation is an extremely accurate classification of the existing mortar pattern and 3D geometry. Although this initial process was completely manual, when compared to traditional drafting techniques, the time spent was not significant and the accuracy achieved is much greater.

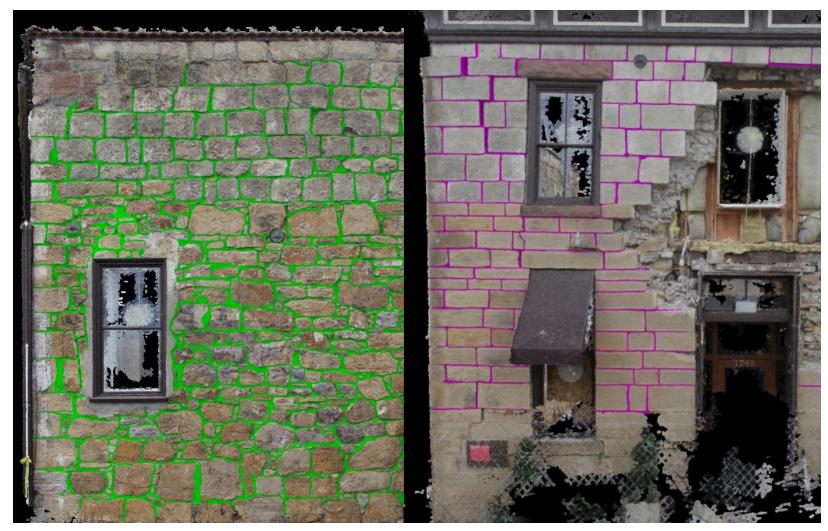

Figure 3. Ground truth tagging of the mortar

Next, a semi-automatic JavaScript function was developed and tested in order to make annotation of the mortar easier for the used by adding some intelligence to the interactive brush. The initial iteration of the mortar brush utilizes the color and normal of the points in order to make decisions based on local statistics. For each bundle of points located in the selective sphere of the brush, statistics of the color and normal are computed. This method assumes that the center of the initial brush click is what the user desires to be tagged. From there, the visualization system steps out from the center point by point and compares the normal and color of the current point to the color and normal of the center point of the initial selection. Based on the color statistics and an allowable variance in the angle of the normal, the script decides if the current point belongs to stone or mortar.

Through testing, initial parameters were chosen so that mortar was classified if the color and normal thresholds were both satisfied. For the color constraint, the current point must be within three standard deviations from the initial point color, based on the local neighborhood statistics. Given the initial reference normal direction and a maximum permissible deviation angle, a candidate point is accepted if the angle between its normal and the reference direction is smaller than the threshold deviation angle. In particular, a normal is accepted if its dot product with the reference normal is greater than the cosine of the threshold angle.

As seen in Figure 2, although many points are queried, only the points corresponding to mortar material are tagged due to the thresholding function defined in the JavaScript. This brush was utilized in three areas with the three different construction styles previously mentioned in order to compare the performance of the brush function under varying colors and normal values. Ten minutes was allocated for each test in which the user classified as much mortar material as possible with the scripted brush. The real-time feedback of the visualization system, which colors the tagged points, allows the user to revisit areas that have not been sufficiently tagged.

The resulting annotated mortar points, seen in Figure 4, were then compared with the ground truth data in order to identify false positive and false negative classifications. The overall results are summarized in Figure 5 detailing the percentages of false positives and negatives as well as the average distance of each false to a classified positive. The last distance comparison is to qualify how far off these false classifications were. For all of the tests, fifty percent of the misclassified points were less than nine millimeters away from a true classified point. This is to say that though the percentages may seem high, the accuracy of the classification was still acceptable. Also to be noted is the comparison in time where each of the three tests was executed in just ten minutes whereas the initial ground truth classification took two and a half hours. 

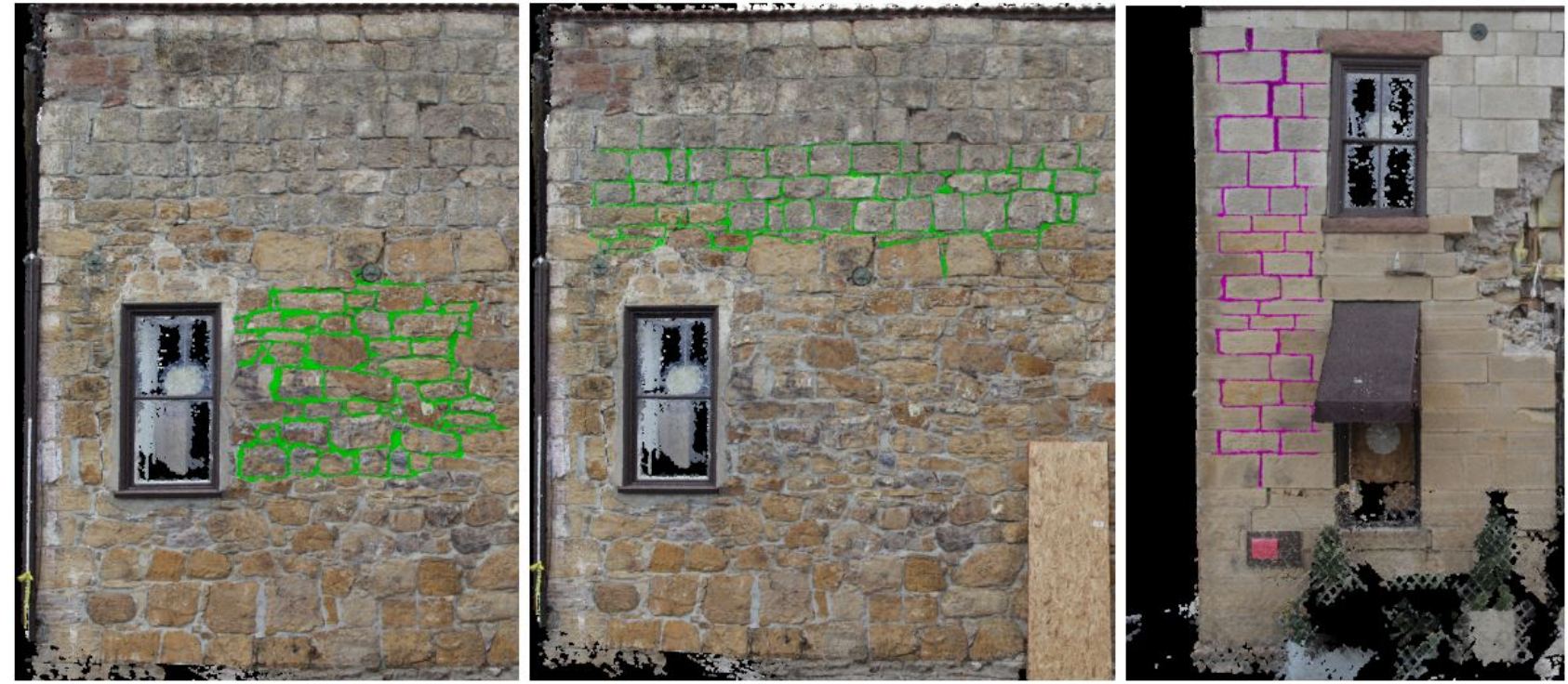

Figure 4. Example of the interactive annotation used on dense point cloud

\begin{tabular}{|l|c|c|c|c|}
\hline \multicolumn{1}{|c|}{ Test } & $\begin{array}{c}\text { False } \\
\text { Positives } \\
\text { (FP) }\end{array}$ & $\begin{array}{c}\text { False } \\
\text { Negatives } \\
\text { (FN) }\end{array}$ & $\begin{array}{c}\text { Avg. distance of } \\
\text { FP from true } \\
\text { positive }\end{array}$ & $\begin{array}{c}\text { Avg. distance of } \\
\text { FN from tagged } \\
\text { positive }\end{array}$ \\
\hline Lower side & $26 \%$ & $30 \%$ & $0.0102 \mathrm{~m}$ & $0.0155 \mathrm{~m}$ \\
\hline Upper side & $23 \%$ & $53 \%$ & $0.0119 \mathrm{~m}$ & $0.0137 \mathrm{~m}$ \\
\hline Front & $46 \%$ & $28 \%$ & $0.0095 \mathrm{~m}$ & $0.0106 \mathrm{~m}$ \\
\hline
\end{tabular}

Figure 5. Ground truth tagging of the mortar

\section{CONCLUSIONS}

The initial implementation of the presented semi-automated interactive classification of masonry point clouds was effective in speeding up the process with limited repercussions in terms of accuracy. Improvements will be made in the future to minimize the amount of false positives classified in the process as well as decrease the amount of false negatives that are not classified along the way. Future efforts will be directed towards the integration of other methods and additional point attributes such as laser intensity when handling point clouds generating through laser scanning techniques. The flexibility of the scripting framework and its integration with the point cloud visualization system will have much broader implications and the presented case study is relatively simple example of the utility of such a robust system.

\section{ACKNOWLEDGEMENTS (OPTIONAL)}

This work was supported by the National Science Foundation under award \#DGE-0966375, "Training, Research and Education in Engineering for Cultural Heritage Diagnostics," and award \#CNS-1338192, "MRI: Development of Advanced Visualization Instrumentation for the Collaborative Exploration of Big Data." Additional support was provided by the Kinsella Fund, the Qualcomm Institute at UC San Diego, the Friends of CISA3, and the World Cultural Heritage Society. Opinions, findings, and conclusions from this study are those of the authors and do not necessarily reflect the opinions of the research sponsors.

\section{REFERENCES}

Crespi, P., Franchi, A., Ronca, P., Giordano, N., Scamardo, M., Gusmeroli, G. and Schiantarelli, G., 2015. From bim to fem: the analysis of an historical masonry building. WIT Transactions on The Built Environment 149, pp. 581-592.

Del Pozo, S., Herrero-Pascual, J., Felipe-García, B., HernándezLópez, D., Rodríguez-Gonzálvez, P. and González-Aguilera, D., 2015. Multi-sensor radiometric study to detect pathologies in historical buildings. The International Archives of Photogrammetry, Remote Sensing and Spatial Information Sciences 40(5), pp. 193.

Dimitrov, A. and Golparvar-Fard, M., 2014. Vision-based material recognition for automated monitoring of construction progress and generating building information modeling from unordered site image collections. Advanced Engineering Informatics 28(1), pp. 37-49.

Dore, C. and Murphy, M., 2013. Semi-automatic modelling of building facades with shape grammars using historic building information modelling. ISPRS International Archives of Photogrammetry, Remote Sensing and Spatial Information Science 40 , pp. 5.

Guarnieri, A., Vettore, A., El-Hakim, S. and Gonzo, L., 2004. Digital photogrammetry and laser scanning in cultural heritage survey. The International Archives of the Photogrammetry, Remote Sensing and Spatial Information Sciences 35, pp. B5.

Guidi, G., Russo, M. and Angheleddu, D., 2014. 3d survey and virtual reconstruction of archeological sites. Digital Applications in Archaeology and Cultural Heritage 1(2), pp. 55-69.

Hess, M., Petrovic, V., Meyer, D., Rissolo, D. and Kuester, F., 2015. Fusion of multimodal three-dimensional data for comprehensive digital documentation of cultural heritage sites. In: 2015 Digital Heritage, Vol. 2, IEEE, pp. 595-602.

Ikeuchi, K., Nakazawa, A., Hasegawa, K. and Ohishi, T., 2003. The great buddha project: Modeling cultural heritage for vr systems through observation. In: Proceedings of the 2nd IEEE/ACM International Symposium on Mixed and Augmented Reality, IEEE Computer Society, p. 7.

Murphy, M., McGovern, E. and Pavia, S., 2009. Historic building information modelling (hbim). Structural Survey 27(4), pp. 311327. 
Murphy, M., McGovern, E. and Pavia, S., 2013. Historic building information modelling-adding intelligence to laser and image based surveys of european classical architecture. ISPRS journal of photogrammetry and remote sensing 76, pp. 89-102.

Oreni, D., Brumana, R., Della Torre, S., Banfi, F. and Previtali, M., 2014. Survey turned into hbim: the restoration and the work involved concerning the basilica di collemaggio after the earthquake (l'aquila). ISPRS Annals of the Photogrammetry, Remote Sensing and Spatial Information Sciences 2(5), pp. 267.

Pesci, A., Bonali, E., Galli, C. and Boschi, E., 2012. Laser scanning and digital imaging for the investigation of an ancient building: Palazzo daccursio study case (bologna, italy). Journal of Cultural Heritage 13(2), pp. 215-220.

Petrovic, V., Gidding, A., Wypych, T., Kuester, F., DeFanti, T. A. and Levy, T. E., 2011. Dealing with archaeology's data avalanche. Computer (7), pp. 56-60.

Petrovic, V., Vanoni, D. J., Richter, A. M., Levy, T. E. and Kuester, F., 2014. Visualizing high resolution three-dimensional and two-dimensional data of cultural heritage sites. Mediterranean Archaeology and Archaeometry 14(4), pp. 93-100.

Quattrini, R., Malinverni, E., Clini, P., Nespeca, R. and Orlietti, E., 2015. From tls to hbim. high quality semantically-aware $3 \mathrm{~d}$ modeling of complex architecture. The International Archives of Photogrammetry, Remote Sensing and Spatial Information Sciences 40(5), pp. 367.

Riveiro, B., Lourenço, P. B., Oliveira, D. V., González-Jorge, H. and Arias, P., 2016. Automatic morphologic analysis of quasiperiodic masonry walls from lidar. Computer-Aided Civil and Infrastructure Engineering 31(4), pp. 305-319.

Sithole, G., 2008. Detection of bricks in a masonry wall. The International Archives of the Photogrammetry, Remote Sensing and Spatial Information Sciences pp. 1-6.

Volk, R., Stengel, J. and Schultmann, F., 2014. Building information modeling (bim) for existing buildingsliterature review and future needs. Automation in construction 38, pp. 109-127. 